\title{
Who knows why regulatory T cells are defective in RA ... IDO
}

4

thas been known for a number of years that regulatory $\mathrm{T}\left(\mathrm{T}_{\mathrm{REG}}\right)$ cells, which normally control excessive immune responses, are defective in patients with rheumatoid arthritis (RA)," says Richard Williams (Kennedy Institute, the University of Oxford) regarding the motivation for his new publication in the journal Arthritis \& Rheumatology. His team of UK researchers has discovered that methylation of the CTLA4 promoter in $\mathrm{T}_{\mathrm{REG}}$ cells prevents antigen-presenting cells (APCs) from expressing indoleaminepyrrole 2,3-dioxygenase (IDO). This epigenetic regulation of CTLA4 is thereby a mechanism to explain defective $\mathrm{T}_{\mathrm{REG}}$-cell function in the pathogenesis of autoimmune diseases, such as RA.

$\mathrm{T}_{\mathrm{REG}}$ cells are thought to prevent the autoreactive T-cell-mediated inflammation that is characteristic of many autoimmune diseases. Patients with RA are known to have $\mathrm{T}_{\text {REG }}$ cells with low surface expression of cytotoxic T lymphocyte protein 4 (CTLA-4), an inhibitory molecule that is vital for the direct cell-cell suppressive function of $\mathrm{T}_{\mathrm{REG}}$ cells. Low CTLA4 expression was therefore thought to be involved in the pathogenesis of synovial inflammation, but no mechanism for this defect had been defined, until now.

By interacting with the co-stimulatory molecules CD80 and CD86 on the surface of cognate APCs, the $\mathrm{T}_{\mathrm{REG}}$ cell protein CTLA-4 is thought to induce expression of IDO1. IDO can then catalyze the conversion of L-tryptophan to $N$-formylkynurenine, a metabolite that feeds into the kynurenine pathway. Kynurenine is an immunotoxin with multiple functions, including the regulation of immune responses, and is implicated in Alzheimer disease and cardiovascular disease. Williams claims, "overexpression of IDO is a mechanism by which tumours escape immune surveillance. However, this is the first report to show that reduced expression of IDO is a potential contributor to the pathogenesis of autoimmunity."

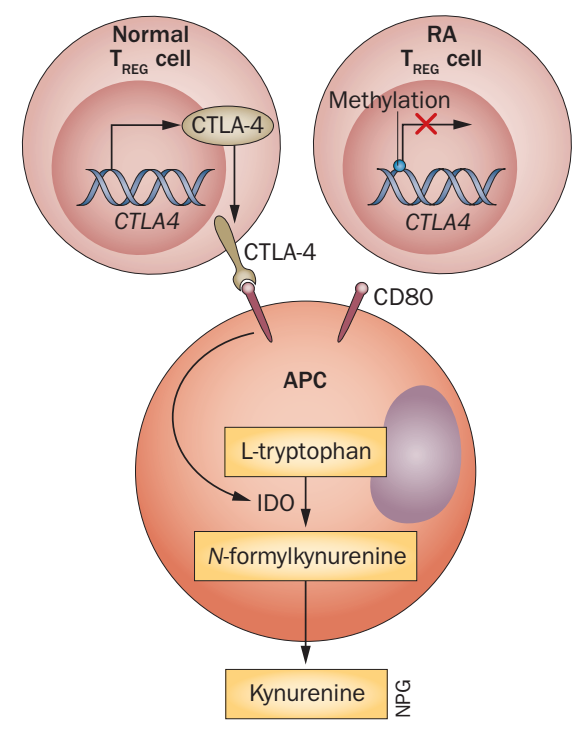

Because the master $\mathrm{T}_{\mathrm{REG}}$ cell transcriptional regulator, FOXP3, is epigenetically regulated, lead researcher on the team, Adam Cribbs (Kennedy Institute, the University of Oxford), explains, "we decided to investigate whether epigenetic changes could account for the reduced functional capacity of $\mathrm{T}_{\mathrm{REG}}$ cells in RA," and says they "focused their attention on methylation of the CTLA4 promoter."

To unravel the mechanism of IDOdependent defective $\mathrm{T}_{\mathrm{REG}}$-cell function in RA, the researchers used flow cytometry to sort effector $\mathrm{T}$ cells $\left(\mathrm{CD} 25^{-} \mathrm{CD} 127^{+}\right)$and natural $\mathrm{T}_{\mathrm{REG}}$ cells $\left(\mathrm{CD} 25^{+} \mathrm{CD} 127^{-}\right)$from peripheral blood of healthy individuals and patients with RA. Importantly, neither group had prior exposure to DMARDs or biologic agents. Although the expression of FoxP3 and also the percentage of $\mathrm{T}_{\mathrm{REG}}$ cells from healthy individuals and patients with RA was not different, autologous suppression assays showed that $T_{\text {REG }}$ cells from patients with RA were defective in suppressing effector T-cell proliferation and IFN- $\gamma$ production.

The researchers also confirmed the expected low surface-expression of CTLA- 4 by $\mathrm{T}_{\mathrm{REG}}$ cells from patients with $\mathrm{RA}$, and with an actinomycin D chase assay then showed that low expression is a consequence of reduced transcriptional activity, not mRNA instability.

Having established the transcriptional defect, they analysed the methylation of CpG residues in the CTLA4 promoter region. Methylation -658 residues from the ATG start codon was substantially higher in $\mathrm{T}_{\mathrm{REG}}$ cells from patients with RA than from healthy individuals.

By ChIP assay, a novel nuclear factor of activated T cells (NFAT)-binding site was then identified in the vicinity of the $-658 \mathrm{CpG}$. The researchers concluded that CTLA4 expression is reduced in $\mathrm{T}_{\text {REG }}$ cells from patients with RA because methylation of its promoter region inhibits NFATc1 binding.

Importantly, neutralization of CTLA-4 in vitro prevented $\mathrm{T}_{\mathrm{REG}}$ cells isolated from healthy donors from suppressing autologous effector $\mathrm{T}$ cells and decreased kynurenine production as measured by high-performance liquid chromatography. Reintroduction of CTLA-4-Ig into the autologous suppression assay cultures reversed this effect, confirming that $\mathrm{T}_{\mathrm{REG}}$ cells from patients with RA are defective in upregulating the IDO pathway as a result of epigenetic repression of CTLA4. Williams says these CTLA-4-Ig data could even explain "a possible mode of action of abatacept in RA."

Exactly how or why CTLA4 methylation is altered in patients with RA is still unknown and is an area for further investigation. Williams says, for now, his team are trying to understand "whether the conclusions drawn from the study are specific for RA, or if they may apply to other autoimmune diseases." In the future he hopes they will "identify environmental and other factors that account for this change in CTLA4 methylation".

Nicholas J. Bernard

Original article Cribbs, A.P. et al. Regulatory T cell function in rheumatoid arthritis is compromised by CTLA-4 promoter methylation resulting in a failture to activate the IDO pathway. Arthritis Rheum. doi:10.1002/art.38715 